\title{
Multi-Style Generative Reading Comprehension
}

\author{
Kyosuke Nishida ${ }^{1}$, Itsumi Saito ${ }^{1}$, Kosuke Nishida ${ }^{1}$, \\ Kazutoshi Shinoda ${ }^{2 *}$, Atsushi Otsuka ${ }^{1}$, Hisako Asano ${ }^{1}$, Junji Tomita $^{1}$ \\ ${ }^{1}$ NTT Media Intelligence Laboratory, NTT Corporation $\quad{ }^{2}$ The University of Tokyo \\ kyosuke.nishida@acm.org
}

\begin{abstract}
This study tackles generative reading comprehension (RC), which consists of answering questions based on textual evidence and natural language generation (NLG). We propose a multi-style abstractive summarization model for question answering, called Masque. The proposed model has two key characteristics. First, unlike most studies on RC that have focused on extracting an answer span from the provided passages, our model instead focuses on generating a summary from the question and multiple passages. This serves to cover various answer styles required for real-world applications. Second, whereas previous studies built a specific model for each answer style because of the difficulty of acquiring one general model, our approach learns multi-style answers within a model to improve the NLG capability for all styles involved. This also enables our model to give an answer in the target style. Experiments show that our model achieves state-of-the-art performance on the Q\&A task and the Q\&A + NLG task of MS MARCO 2.1 and the summary task of NarrativeQA. We observe that the transfer of the style-independent NLG capability to the target style is the key to its success.
\end{abstract}

\section{Introduction}

Question answering has been a long-standing research problem. Recently, reading comprehension (RC), a challenge to answer a question given textual evidence provided in a document set, has received much attention. Current mainstream studies have treated RC as a process of extracting an answer span from one passage (Rajpurkar et al., 2016, 2018) or multiple passages (Joshi et al., 2017; Yang et al., 2018), which is usually done by predicting the start and end positions of the answer (Yu et al., 2018; Devlin et al., 2018).

\footnotetext{
${ }^{*}$ Work done during an internship at NTT.
}

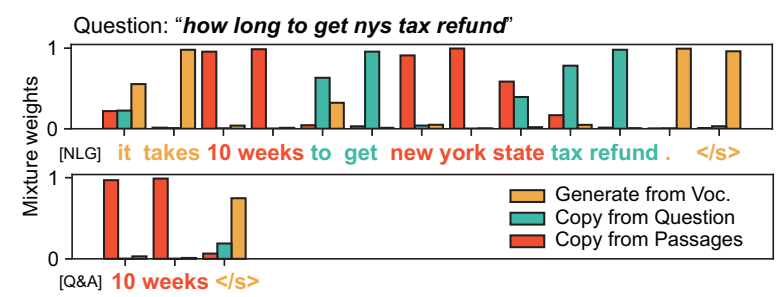

Figure 1: Visualization of how our model generates an answer on MS MARCO. Given an answer style (top: NLG, bottom: Q\&A), the model controls the mixture of three distributions for generating words from a vocabulary and copying words from the question and multiple passages at each decoding step.

The demand for answering questions in natural language is increasing rapidly, and this has led to the development of smart devices such as Alexa. In comparison with answer span extraction, however, the natural language generation (NLG) capability for RC has been less studied. While datasets such as MS MARCO (Bajaj et al., 2018) and NarrativeQA (Kociský et al., 2018) have been proposed for providing abstractive answers, the stateof-the-art methods for these datasets are based on answer span extraction (Wu et al., 2018; Hu et al., 2018). Generative models suffer from a dearth of training data to cover open-domain questions.

Moreover, to satisfy various information needs, intelligent agents should be capable of answering one question in multiple styles, such as wellformed sentences, which make sense even without the context of the question and passages, and concise phrases. These capabilities complement each other, but previous studies cannot use and control different styles within a model.

In this study, we propose Masque, a generative model for multi-passage RC. It achieves stateof-the-art performance on the Q\&A task and the Q\&A + NLG task of MS MARCO 2.1 and the summary task of NarrativeQA. The main contri- 
butions of this study are as follows.

Multi-source abstractive summarization. We introduce the pointer-generator mechanism (See et al., 2017) for generating an abstractive answer from the question and multiple passages, which covers various answer styles. We extend the mechanism to a Transformer (Vaswani et al., 2017) based one that allows words to be generated from a vocabulary and to be copied from the question and passages.

Multi-style learning for style control and transfer. We introduce multi-style learning that enables our model to control answer styles and improves RC for all styles involved. We also extend the pointer-generator to a conditional decoder by introducing an artificial token corresponding to each style, as in (Johnson et al., 2017). For each decoding step, it controls the mixture weights over three distributions with the given style (Figure 1).

\section{Problem Formulation}

This paper considers the following task:

PROBLEM 1. Given a question with $J$ words $x^{q}=$ $\left\{x_{1}^{q}, \ldots, x_{J}^{q}\right\}$, a set of $K$ passages, where the $k$-th passage is composed of $L$ words $x^{p_{k}}=$ $\left\{x_{1}^{p_{k}}, \ldots, x_{L}^{p_{k}}\right\}$, and an answer style label $s$, an RC model outputs an answer $y=\left\{y_{1}, \ldots, y_{T}\right\}$ conditioned on the style.

In short, given a 3-tuple $\left(x^{q},\left\{x^{p_{k}}\right\}, s\right)$, the system predicts $P(y)$. The training data is a set of 6-tuples: $\left(x^{q},\left\{x^{p_{k}}\right\}, s, y, a,\left\{r^{p_{k}}\right\}\right)$, where $a$ and $\left\{r^{p_{k}}\right\}$ are optional. Here, $a$ is 1 if the question is answerable with the provided passages and 0 otherwise, and $r^{p_{k}}$ is 1 if the $k$-th passage is required to formulate the answer and 0 otherwise.

\section{Proposed Model}

We propose a Multi-style Abstractive Summarization model for QUEstion answering, called Masque. Masque directly models the conditional probability $p\left(y \mid x^{q},\left\{x^{p_{k}}\right\}, s\right)$. As shown in Figure 2 , it consists of the following modules.

1. The question-passages reader ( $\$ 3.1$ ) models interactions between the question and passages.

2. The passage ranker ( $(3.2)$ finds passages relevant to the question.

3. The answer possibility classifier ( $§ 3.3)$ identifies answerable questions.

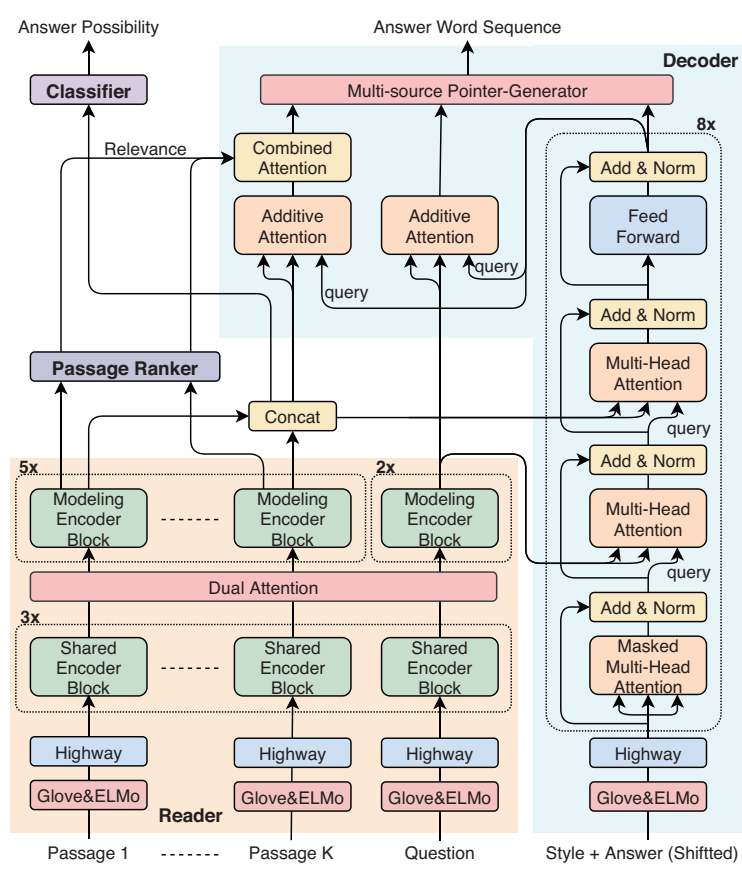

Figure 2: Masque model architecture.

4. The answer sentence decoder (§3.4) outputs an answer sentence conditioned on the target style.

Our model is based on multi-source abstractive summarization: the answer that it generates can be viewed as a summary from the question and passages. The model also learns multi-style answers together. With these two characteristics, we aim to acquire the style-independent NLG ability and transfer it to the target style. In addition, to improve natural language understanding in the reader module, our model considers RC, passage ranking, and answer possibility classification together as multi-task learning.

\subsection{Question-Passages Reader}

The reader module is shared among multiple answer styles and the three task-specific modules.

\subsubsection{Word Embedding Layer}

Let $x^{q}$ and $x^{p_{k}}$ represent one-hot vectors (of size $V)$ for words in the question and the $k$-th passage. First, this layer projects each of the vectors to a $d_{\text {word-dimensional vector with a pre- }}$ trained weight matrix $W^{e} \in \mathbb{R}^{d_{\text {word }} \times V}$ such as GloVe (Pennington et al., 2014). Next, it uses contextualized word representations via ELMo (Peters et al., 2018), which allows our model to use morphological clues to form robust representations for out-of-vocabulary words unseen in training. Then, the concatenation of the word and con- 
textualized vectors is passed to a two-layer highway network (Srivastava et al., 2015) to fuse the two types of embeddings, as in (Seo et al., 2017). The highway network is shared by the question and passages.

\subsubsection{Shared Encoder Layer}

This layer uses a stack of Transformer blocks, which are shared by the question and passages, on top of the embeddings provided by the word embedding layer. The input of the first block is immediately mapped to a $d$-dimensional vector by a linear transformation. The outputs of this layer are $E^{p_{k}} \in \mathbb{R}^{d \times L}$ for each $k$-th passage, and $E^{q} \in \mathbb{R}^{d \times J}$ for the question.

Transformer encoder block. The block consists of two sub-layers: a self-attention layer and a position-wise feed-forward network. For the self-attention layer, we adopt the multi-head attention mechanism (Vaswani et al., 2017). Following GPT (Radford et al., 2018), the feed-forward network consists of two linear transformations with a GELU (Hendrycks and Gimpel, 2016) activation function in between. Each sub-layer is placed inside a residual block (He et al., 2016). For an input $x$ and a given sub-layer function $f$, the output is $\mathrm{LN}(f(x)+x)$, where $\mathrm{LN}$ indicates the layer normalization (Ba et al., 2016). To facilitate these residual connections, all sub-layers produce a sequence of $d$-dimensional vectors. Note that our model does not use any position embeddings in this block because ELMo gives the positional information of the words in each sequence.

\subsubsection{Dual Attention Layer}

This layer uses a dual attention mechanism to fuse information from the question to the passages as well as from the passages to the question.

It first computes a similarity matrix $U^{p_{k}} \in$ $\mathbb{R}^{L \times J}$ between the question and the $k$-th passage, as done in (Seo et al., 2017), where

$$
U_{l j}^{p_{k}}=w^{a \top}\left[E_{l}^{p_{k}} ; E_{j}^{q} ; E_{l}^{p_{k}} \odot E_{j}^{q}\right]
$$

indicates the similarity between the $l$-th word of the $k$-th passage and the $j$-th question word. The $w^{a} \in \mathbb{R}^{3 d}$ are learnable parameters. The $\odot$ operator denotes the Hadamard product, and the [;] operator denotes vector concatenation across the rows. Next, the layer obtains the row and column normalized similarity matrices $A^{p_{k}}=$ $\operatorname{softmax}_{j}\left(U^{p_{k} \top}\right)$ and $B^{p_{k}}=\operatorname{softmax}_{l}\left(U^{p_{k}}\right)$. It then uses DCN (Xiong et al., 2017) to obtain dual attention representations, $G^{q \rightarrow p_{k}} \in \mathbb{R}^{5 d \times L}$ and $G^{p \rightarrow q} \in \mathbb{R}^{5 d \times J}$ :

$$
\begin{aligned}
G^{q \rightarrow p_{k}} & =\left[E^{p_{k}} ; \bar{A}^{p_{k}} ; \overline{\bar{A}}^{p_{k}} ; E^{p_{k}} \odot \bar{A}^{p_{k}} ; E^{p_{k}} \odot \overline{\bar{A}}^{p_{k}}\right] \\
G^{p \rightarrow q} & =\left[E^{q} ; \bar{B} ; \overline{\bar{B}} ; E^{q} \odot \bar{B} ; E^{q} \odot \overline{\bar{B}}\right] .
\end{aligned}
$$

Here, $\bar{A}^{p_{k}}=E^{q} A^{p_{k}}, \bar{B}^{p_{k}}=E^{p_{k}} B^{p_{k}}, \overline{\bar{A}}^{p_{k}}=$ $\bar{B}^{p_{k}} A^{p_{k}}, \overline{\bar{B}}^{p_{k}}=\bar{A}^{p_{k}} B^{p_{k}}, \bar{B}=\max _{k}\left(\bar{B}^{p_{k}}\right)$, and $\overline{\bar{B}}=\max _{k}\left(\overline{\bar{B}}^{p_{k}}\right)$.

\subsubsection{Modeling Encoder Layer}

This layer uses a stack of the Transformer encoder blocks for question representations and obtains $M^{q} \in \mathbb{R}^{d \times J}$ from $G^{p \rightarrow q}$. It also uses another stack for passage representations and obtains $M^{p_{k}} \in \mathbb{R}^{d \times L}$ from $G^{q \rightarrow p_{k}}$ for each $k$-th passage. The outputs of this layer, $M^{q}$ and $\left\{M^{p_{k}}\right\}$, are passed on to the answer sentence decoder; the $\left\{M^{p_{k}}\right\}$ are also passed on to the passage ranker and the answer possibility classifier.

\subsection{Passage Ranker}

The ranker maps the output of the modeling layer, $\left\{M^{p_{k}}\right\}$, to the relevance score of each passage. It takes the output for the first word, $M_{1}^{p_{k}}$, which corresponds to the beginning-of-sentence token, to obtain the aggregate representation of each passage sequence. Given $w^{r} \in \mathbb{R}^{d}$ as learnable parameters, it calculates the relevance of each $k$-th passage to the question as

$$
\beta^{p_{k}}=\operatorname{sigmoid}\left(w^{r \top} M_{1}^{p_{k}}\right) .
$$

\subsection{Answer Possibility Classifier}

The classifier maps the output of the modeling layer to a probability for the answer possibility. It also takes the output for the first word, $M_{1}^{p_{k}}$, for all passages and concatenates them. Given $w^{c} \in \mathbb{R}^{K d}$ as learnable parameters, it calculates the answer possibility for the question as

$$
P(a)=\operatorname{sigmoid}\left(w^{c \top}\left[M_{1}^{p_{1}} ; \ldots ; M_{1}^{p_{K}}\right]\right) .
$$

\subsection{Answer Sentence Decoder}

Given the outputs provided by the reader module, the decoder generates a sequence of answer words one element at a time. It is autoregressive (Graves, 2013), consuming the previously generated words as additional input at each decoding step. 


\subsubsection{Word Embedding Layer}

Let $y$ represent one-hot vectors of the words in the answer. This layer has the same components as the word embedding layer of the reader module, except that it uses a unidirectional ELMo to ensure that the predictions for position $t$ depend only on the known outputs at positions previous to $t$.

Artificial tokens. To be able to use multiple answer styles within a single system, our model introduces an artificial token corresponding to the style at the beginning of the answer $\left(y_{1}\right)$, as done in (Johnson et al., 2017; Takeno et al., 2017). At test time, the user can specify the first token to control the style. This modification does not require any changes to the model architecture. Note that introducing the token at the decoder prevents the reader module from depending on the answer style.

\subsubsection{Attentional Decoder Layer}

This layer uses a stack of Transformer decoder blocks on top of the embeddings provided by the word embedding layer. The input is immediately mapped to a $d$-dimensional vector by a linear transformation, and the output is a sequence of $d$-dimensional vectors: $\left\{s_{1}, \ldots, s_{T}\right\}$.

Transformer decoder block. In addition to the encoder block, this block consists of the second and third sub-layers after the self-attention block and before the feed-forward network, as shown in Figure 2. As in (Vaswani et al., 2017), the selfattention sub-layer uses a sub-sequent mask to prevent positions from attending to subsequent positions. The second and third sub-layers perform the multi-head attention over $M^{q}$ and $M^{p_{\text {all }}}$, respectively. The $M^{p_{\text {all }}}$ is the concatenated outputs of the encoder stack for the passages,

$$
M^{p_{\text {all }}}=\left[M^{p_{1}}, \ldots, M^{p_{K}}\right] \in \mathbb{R}^{d \times K L} .
$$

Here, the [,] operator denotes vector concatenation across the columns. This attention for the concatenated passages produces attention weights that are comparable between passages.

\subsubsection{Multi-source Pointer-Generator}

Our extended mechanism allows both words to be generated from a vocabulary and words to be copied from both the question and multiple passages (Figure 3). We expect that the capability of copying words will be shared among answer styles.

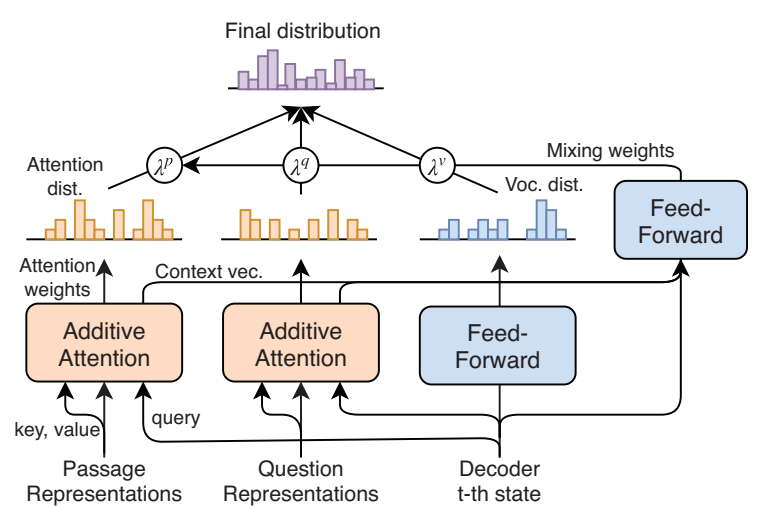

Figure 3: Multi-source pointer-generator mechanism. For each decoding step $t$, mixture weights $\lambda^{v}, \lambda^{q}, \lambda^{p}$ for the probability of generating words from the vocabulary and copying words from the question and the passages are calculated. The three distributions are weighted and summed to obtain the final distribution.

Extended vocabulary distribution. Let the extended vocabulary, $V_{\text {ext }}$, be the union of the common words (a small subset of the full vocabulary, $V$, defined by the input-side word embedding matrix) and all words appearing in the input question and passages. $P^{v}$ then denotes the probability distribution of the $t$-th answer word, $y_{t}$, over the extended vocabulary. It is defined as:

$$
P^{v}\left(y_{t}\right)=\operatorname{softmax}\left(W^{2^{\top}}\left(W^{1} s_{t}+b^{1}\right)\right),
$$

where the output embedding $W^{2} \in \mathbb{R}^{d_{\text {word }} \times V_{\text {ext }} \text { is }}$ tied with the corresponding part of the input embedding (Inan et al., 2017), and $W^{1} \in \mathbb{R}^{d_{\text {word }} \times d}$ and $b^{1} \in \mathbb{R}^{d_{\text {word }}}$ are learnable parameters. $P^{v}\left(y_{t}\right)$ is zero if $y_{t}$ is an out-of-vocabulary word for $V$.

Copy distributions. A recent Transformerbased pointer-generator randomly chooses one of the attention-heads to form a copy distribution; that approach gave no significant improvements in text summarization (Gehrmann et al., 2018).

In contrast, our model uses an additional attention layer for each copy distribution on top of the decoder stack. For the passages, the layer takes $s_{t}$ as the query and outputs $\alpha_{t}^{p} \in \mathbb{R}^{K L}$ as the attention weights and $c_{t}^{p} \in \mathbb{R}^{d}$ as the context vectors:

$$
\begin{aligned}
e_{l}^{p_{k}} & =w^{p \top} \tanh \left(W^{p m} M_{l}^{p_{k}}+W^{p s} s_{t}+b^{p}\right), \\
\alpha_{t}^{p} & =\operatorname{softmax}\left(\left[e^{p_{1}} ; \ldots ; e^{p_{K}}\right]\right), \\
c_{t}^{p} & =\sum_{l} \alpha_{t l}^{p} M_{l}^{p_{\text {all }}},
\end{aligned}
$$

where $w^{p}, b^{p} \in \mathbb{R}^{d}$ and $W^{p m}, W^{p s} \in \mathbb{R}^{d \times d}$ are learnable parameters. For the question, our model 
uses another identical layer and obtains $\alpha_{t}^{q} \in \mathbb{R}^{J}$ and $c_{t}^{q} \in \mathbb{R}^{d}$. As a result, $P^{q}$ and $P^{p}$ are the copy distributions over the extended vocabulary:

$$
\begin{aligned}
& P^{q}\left(y_{t}\right)=\sum_{j: x_{j}^{q}=y_{t}} \alpha_{t j}^{q}, \\
& P^{p}\left(y_{t}\right)=\sum_{l: x_{l}^{p} k_{k(l)}=y_{t}} \alpha_{t l}^{p},
\end{aligned}
$$

where $k(l)$ means the passage index corresponding to the $l$-th word in the concatenated passages.

Final distribution. The final distribution of $y_{t}$ is defined as a mixture of the three distributions:

$$
\begin{aligned}
P\left(y_{t}\right) & =\lambda^{v} P^{v}\left(y_{t}\right)+\lambda^{q} P^{q}\left(y_{t}\right)+\lambda^{p} P^{p}\left(y_{t}\right), \\
\lambda^{v}, \lambda^{q}, \lambda^{p} & =\operatorname{softmax}\left(W^{m}\left[s_{t} ; c_{t}^{q} ; c_{t}^{p}\right]+b^{m}\right),
\end{aligned}
$$

where $W^{m} \in \mathbb{R}^{3 \times 3 d}$ and $b^{m} \in \mathbb{R}^{3}$ are learnable parameters.

\subsubsection{Combined Attention}

In order not to attend words in irrelevant passages, our model introduces a combined attention. While the original technique combined word and sentence level attentions (Hsu et al., 2018), our model combines the word and passage level attentions. The word attention, Eq. 1, is re-defined as

$$
\alpha_{t l}^{p}=\frac{\alpha_{t l}^{p} \beta^{p_{k(l)}}}{\sum_{l^{\prime}} \alpha_{t l^{\prime}}^{p} \beta^{p_{k\left(l^{\prime}\right)}}} .
$$

\subsection{Loss Function}

We define the training loss as the sum of losses via

$$
L(\theta)=L_{\mathrm{dec}}+\gamma_{\mathrm{rank}} L_{\mathrm{rank}}+\gamma_{\mathrm{cls}} L_{\mathrm{cls}}
$$

where $\theta$ is the set of all learnable parameters, and $\gamma_{\text {rank }}$ and $\gamma_{\mathrm{cls}}$ are balancing parameters.

The loss of the decoder, $L_{\mathrm{dec}}$, is the negative log likelihood of the whole target answer sentence averaged over $N_{\text {able }}$ answerable examples:

$$
L_{\mathrm{dec}}=-\frac{1}{N_{\mathrm{able}}} \sum_{(a, y) \in \mathcal{D}} \frac{a}{T} \sum_{t} \log P\left(y_{t}\right),
$$

where $\mathcal{D}$ is the training dataset. The losses of the passage ranker, $L_{\text {rank }}$, and the answer possibility classifier, $L_{\mathrm{cls}}$, are the binary cross entropy between the true and predicted values averaged over all $N$ examples:

$$
\begin{gathered}
L_{\mathrm{rank}}=-\frac{1}{N K} \sum_{k} \sum_{r^{p_{k}} \in \mathcal{D}}\left(\begin{array}{l}
r^{p_{k}} \log \beta^{p_{k}}+ \\
\left(1-r^{p_{k}}\right) \log \left(1-\beta^{p_{k}}\right)
\end{array}\right), \\
L_{\mathrm{cls}}=-\frac{1}{N} \sum_{a \in \mathcal{D}}\left(\begin{array}{l}
a \log P(a)+ \\
(1-a) \log (1-P(a))
\end{array}\right) .
\end{gathered}
$$

\begin{tabular}{c|c|ccc}
\hline Dataset & Subset & Train & Dev. & Eval. \\
\hline & ALL & 808,731 & 101,093 & 101,092 \\
MS MARCO & ANS & 503,370 & 55,636 & - \\
& NLG & 153,725 & 12,467 & - \\
\hline NarrativeQA & Summary & 32,747 & 3,461 & 10,557 \\
\hline
\end{tabular}

Table 1: Numbers of questions used in the experiments.

\section{Experiments on MS MARCO 2.1}

We evaluated our model on MS MARCO 2.1 (Bajaj et al., 2018). It is the sole dataset providing abstractive answers with multiple styles and serves as a great test bed for building open-domain QA agents with the NLG capability that can be used in smart devices. The details of our setup and output examples are in the supplementary material.

\subsection{Setup}

Datasets. MS MARCO 2.1 provides two tasks for generative open-domain QA: the Q\&A task and the Q\&A + Natural Language Generation (NLG) task. Both tasks consist of questions submitted to Bing by real users, and each question refers to ten passages. The dataset also includes annotations on the relevant passages, which were selected by humans to form the final answers, and on whether there was no answer in the passages.

Answer styles. We associated the two tasks with two answer styles. The NLG task requires a wellformed answer that is an abstractive summary of the question and passages, averaging 16.6 words. The Q\&A task also requires an abstractive answer but prefers it to be more concise than in the NLG task, averaging 13.1 words, and many of the answers do not contain the context of the question. For the question "tablespoon in cup", a reference answer in the Q\&A task is "16," while that in the NLG task is "There are 16 tablespoons in a cup."

Subsets. In addition to the ALL dataset, we prepared two subsets for ablation tests as listed in Table 1. The ANS set consisted of answerable questions, and the NLG set consisted of the answerable questions and well-formed answers, so that NLG $\subset$ ANS $\subset$ ALL. We note that multi-style learning enables our model to learn from different answer styles of data (i.e., the ANS set), and multi-task learning with the answer possibility classifier enables our model to learn from both answerable and unanswerable data (i.e., the ALL set).

Training and Inference. We trained our model with mini-batches consisting of multi-style an- 


\begin{tabular}{l|cc|cc}
\hline \multirow{2}{*}{ Model } & \multicolumn{2}{|c|}{ NLG } & \multicolumn{2}{c}{ Q\&A } \\
& R-L & B-1 & R-L & B-1 \\
\hline BiDAF $^{a}$ & 16.91 & 9.30 & 23.96 & 10.64 \\
Deep Cascade QA $^{b}$ & 35.14 & 37.35 & 52.01 & $\mathbf{5 4 . 6 4}$ \\
S-Net+CES2S $^{c}$ & 45.04 & 40.62 & 44.96 & 46.36 \\
BERT+Multi-PGNet $^{d}$ & 47.37 & 45.09 & 48.14 & 52.03 \\
Selector+CCG $^{e}$ & 47.39 & 45.26 & 50.63 & 52.03 \\
VNET $^{f}$ & 48.37 & 46.75 & 51.63 & 54.37 \\
\hline Masque (NLG; single) $^{\text {Masque (NLG; ensemble) }}$ & 49.19 & 49.63 & 48.42 & 48.68 \\
Masque (Q\&A; single) $^{\text {49.61 }}$ & $\mathbf{5 0 . 1 3}$ & 48.92 & 48.75 \\
Masque (Q\&A; ensemble) & 28.53 & 36.62 & 50.93 & 42.37 \\
\hline Human Performance & 63.21 & 53.03 & 53.87 & 48.50 \\
\hline
\end{tabular}

Table 2: Performance of our and competing models on the MS MARCO V2 leaderboard (4 March 2019). ${ }^{a}$ Seo et al. (2017); ${ }^{b}$ Yan et al. (2019); ${ }^{c}$ Shao (unpublished), a variant of Tan et al. (2018); ${ }^{d} \mathrm{Li}$ (unpublished), a model using Devlin et al. (2018) and See et al. (2017); ${ }^{e}$ Qian (unpublished); ${ }^{f} \mathrm{Wu}$ et al. (2018). Whether the competing models are ensemble models or not is unreported.

swers that were randomly sampled. We used a greedy decoding algorithm and did not use any beam search or random sampling, because they did not provide any improvements.

Evaluation metrics and baselines. ROUGE-L and BLEU-1 were used to evaluate the models' $\mathrm{RC}$ performance, where ROUGE-L is the main metric on the official leaderboard. We used the reported scores of extractive (Seo et al., 2017; Yan et al., 2019; Wu et al., 2018), generative (Tan et al., 2018), and unpublished RC models at the submission time.

In addition, to evaluate the individual contributions of our modules, we used MAP and MRR for the ranker and $F_{1}$ for the classifier, where the positive class was the answerable questions.

\subsection{Results}

Does our model achieve state-of-the-art on the two tasks with different styles? Table 2 shows the performance of our model and competing models on the leaderboard. Our ensemble model of six training runs, where each model was trained with the two answer styles, achieved state-of-theart performance on both tasks in terms of ROUGEL. In particular, for the NLG task, our single model outperformed competing models in terms of both ROUGE-L and BLEU-1.

Does multi-style learning improve the NLG performance? Table 3 lists the results of an ablation test for our single model (controlled with

\begin{tabular}{l|c|cc}
\hline Model & Train & R-L & B-1 \\
\hline Masque (NLG style; single) & ALL & $\mathbf{6 9 . 7 7}$ & $\mathbf{6 5 . 5 6}$ \\
w/o multi-style learning $(\S 3.4 .2)$ & NLG & 68.20 & 63.95 \\
$\hookrightarrow$ w/o Transformer $(\S 3.1 .2, \S 3.4 .2)$ & NLG & 67.13 & 62.96 \\
w/o passage ranker $(\S 3.2)$ & NLG & 68.05 & 63.82 \\
w/o possibility classifier $(\S 3.3)$ & ANS & 69.64 & 65.41 \\
\hline Masque w/ gold passage ranker & ALL & 78.70 & 78.14 \\
\hline
\end{tabular}

Table 3: Ablation test results on the NLG dev. set. The models were trained with the subset listed in "Train".

\begin{tabular}{l|c|cc}
\hline Model & Train & MAP & MRR \\
\hline Bing (initial ranking) & - & 34.62 & 35.00 \\
\hline Masque (single) & ALL & $\mathbf{6 9 . 5 1}$ & $\mathbf{6 9 . 9 6}$ \\
w/o answer decoder $(\S 3.4)$ & ALL & 67.03 & 67.49 \\
w/o multi-style learning $(\S 3.4 .2)$ & NLG & 65.51 & 65.59 \\
w/o possibility classifier ( $§ 3.3)$ & ANS & 69.08 & 69.54 \\
\hline
\end{tabular}

Table 4: Passage ranking results on the ANS dev. set.

the NLG style) on the NLG dev. set ${ }^{1}$. Our model trained with both styles outperformed the model trained with the single NLG style. Multi-style learning enabled our model to improve its NLG performance by also using non-sentence answers.

Does the Transformer-based pointer-generator improve the NLG performance? Table 3 shows that our model also outperformed the model that used RNNs and self-attentions instead of Transformer blocks as in MCAN (McCann et al., 2018). Our deep decoder captured the multi-hop interaction among the question, the passages, and the answer better than a single-layer LSTM decoder could.

Does joint learning with the ranker and classifier improve NLG performance? Furthermore, Table 3 shows that our model (jointly trained with the passage ranker and answer possibility classifier) outperformed the model that did not use the ranker and classifier. Joint learning thus had a regularization effect on the question-passages reader.

We also confirmed that the gold passage ranker, which can perfectly predict the relevance of passages, significantly improved the RC performance. Passage ranking will be a key to developing a system that can outperform humans.

Does joint learning improve the passage ranking performance? Table 4 lists the passage ranking performance on the ANS dev. set ${ }^{2}$. The

\footnotetext{
${ }^{1}$ We confirmed with the organizer that the dev. results were much better than the test results, but there was no problem.

${ }^{2}$ This evaluation requires our ranker to re-rank 10 passages. It is not the same as the Passage Re-ranking task.
} 


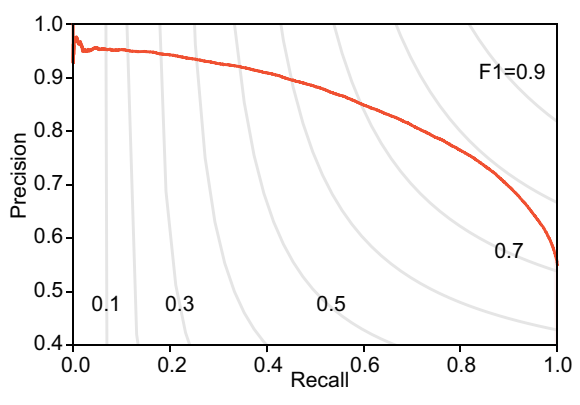

Figure 4: Precision-recall curve for answer possibility classification on the ALL dev. set.

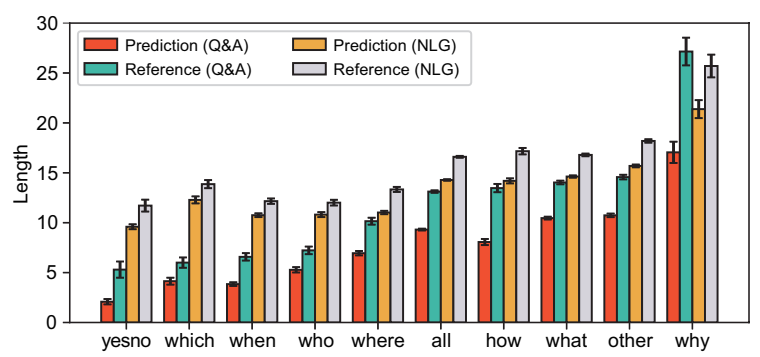

Figure 5: Lengths of answers generated by Masque broken down by the answer style and query type on the NLG dev. set. The error bars indicate standard errors.

ranker shares the question-passages reader with the answer decoder, and this sharing contributed to improvements over the ranker trained without the answer decoder. Also, our ranker outperformed the initial ranking provided by Bing by a significant margin.

Does our model accurately identify answerable questions? Figure 4 shows the precision-recall curve for answer possibility classification on the ALL dev. set. Our model identified the answerable questions well. The maximum $F_{1}$ score was 0.7893 , where the threshold of answer possibility was 0.4411 . This is the first report on answer possibility classification with MS MARCO 2.1.

Does our model control answer lengths with different styles? Figure 5 shows the lengths of the answers generated by our model broken down by the answer style and query type. The generated answers were relatively shorter than the reference answers, especially for the Q\&A task, but well controlled with the target style for every query type. The short answers degraded our model's BLEU scores in the Q\&A task (Table 2) because of BLEU's brevity penalty (Papineni et al., 2002).

\section{Experiments on NarrativeQA}

Next, we evaluated our model on NarrativeQA (Kociský et al., 2018). It requires understanding the underlying narrative rather than relying on shallow pattern matching. Our detailed setup and output examples are in the supplementary material.

\subsection{Setup}

We only describe the settings specific to this experiment.

Datasets. Following previous studies, we used the summary setting for the comparisons with the reported baselines, where each question refers to one summary (averaging 659 words), and there is no unanswerable questions. Our model therefore did not use the passage ranker and answer possibility classifier.

Answer styles. The NarrativeQA dataset does not explicitly provide multiple answer styles. In order to evaluate the effectiveness of multi-style learning, we used the NLG subset of MS MARCO as additional training data. We associated the NarrativeQA and NLG datasets with two answer styles. The answer style of NarrativeQA (NQA) is different from that of MS MARCO (NLG) in that the answers are short (averaging 4.73 words) and contained frequently pronouns. For instance, for the question "Who is Mark Hunter?", a reference is "He is a high school student in Phoenix."

Evaluation metrics and baselines. BLEU-1 and 4, METEOR, and ROUGE-L were used in accordance with the evaluation in the dataset paper (Kociský et al., 2018). We used the reports of top-performing extractive (Seo et al., 2017; Tay et al., 2018; Hu et al., 2018) and generative (Bauer et al., 2018; Indurthi et al., 2018) models.

\subsection{Results}

Does our model achieve state-of-the-art performance? Table 5 shows that our single model, trained with two styles and controlled with the NQA style, pushed forward the state-of-the-art by a significant margin. The evaluation scores of the model controlled with the NLG style were low because the two styles are different. Also, our model without multi-style learning (trained with only the NQA style) outperformed the baselines in terms of ROUGE-L. This indicates that our model architec- 


\begin{tabular}{l|cccc}
\hline Model & $\mathrm{B}-1$ & $\mathrm{~B}-4$ & $\mathrm{M}$ & $\mathrm{R}-\mathrm{L}$ \\
\hline BiDAF $^{a}$ & 33.72 & 15.53 & 15.38 & 36.30 \\
DECAPROP $^{b}$ & 42.00 & 23.42 & 23.42 & 40.07 \\
MHPGM+NOIC $^{c}$ & 43.63 & 21.07 & 19.03 & 44.16 \\
ConZNet $^{d}$ & 42.76 & 22.49 & 19.24 & 46.67 \\
RMR+A2D $^{e}$ & 50.4 & 26.5 & $\mathrm{~N} / \mathrm{A}$ & 53.3 \\
\hline Masque (NQA) $^{\text {w/o multi-style learning }}$ & $\mathbf{5 4 . 1 1}$ & $\mathbf{3 0 . 4 3}$ & $\mathbf{2 6 . 1 3}$ & $\mathbf{5 9 . 8 7}$ \\
Masque (NLG) & 39.70 & 20.98 & 21.95 & 54.74 \\
\hline \hline Masque (NQA; valid.) $^{f}$ & 52.78 & 28.72 & 25.38 & 58.94 \\
\hline
\end{tabular}

Table 5: Performance of our and competing models on the NarrativeQA test set. ${ }^{a}$ Seo et al. (2017); ${ }^{b}$ Tay et al. (2018); ${ }^{c}$ Bauer et al. (2018); ${ }^{d}$ Indurthi et al. (2018); ${ }^{e} \mathrm{Hu}$ et al. (2018). ${ }^{f}$ Results on the NarrativeQA validation set.

ture itself is powerful for natural language understanding in RC.

\section{Related Work and Discussion}

Transfer and multi-task learning in RC. Recent breakthroughs in transfer learning demonstrate that pre-trained language models perform well on RC with minimal modifications (Peters et al., 2018; Devlin et al., 2018; Radford et al., 2018, 2019). In addition, our model also uses ELMo (Peters et al., 2018) for contextualized embeddings.

Multi-task learning is a transfer mechanism to improve generalization performance (Caruana, 1997), and it is generally applied by sharing the hidden layers between all tasks, while keeping task-specific layers. Wang et al. (2018) and Nishida et al. (2018) reported that the sharing of the hidden layers between the multi-passage RC and passage ranking tasks was effective. Our results also showed the effectiveness of the sharing of the question-passages reader module among the $\mathrm{RC}$, passage ranking, and answer possibility classification tasks.

In multi-task learning without task-specific layers, Devlin et al. (2018) and Chen et al. (2017) improved RC performance by learning multiple datasets from the same extractive RC setting. McCann et al. (2018) and Yogatama et al. (2019) investigated multi-task and curriculum learning on many different NLP tasks; their results were below task-specific RC models. Our multi-style learning does not use style-specific layers; instead uses a style-conditional decoder.

Generative RC. S-Net (Tan et al., 2018) used an extraction-then-synthesis mechanism for multi- passage RC. The models proposed by McCann et al. (2018), Bauer et al. (2018), and Indurthi et al. (2018) used an RNN-based pointer-generator mechanism for single-passage RC. Although these mechanisms can alleviate the lack of training data, large amounts of data are still required. Our multistyle learning will be a key technique enabling learning from many RC datasets with different styles.

In addition to MS MARCO and NarrativeQA, there are other datasets that provide abstractive answers. DuReader (He et al., 2018), a Chinese multi-document RC dataset, provides longer documents and answers than those of MS MARCO. DuoRC (Saha et al., 2018) and CoQA (Reddy et al., 2018) contain abstractive answers; most of the answers are short phrases.

Controllable text generation. Many studies have been carried out in the framework of style transfer, which is the task of rephrasing a text so that it contains specific styles such as sentiment. Recent studies have used artificial tokens (Sennrich et al., 2016; Johnson et al., 2017), variational auto-encoders (Hu et al., 2017), or adversarial training (Fu et al., 2018; Tsvetkov et al., 2018) to separate the content and style on the encoder side. On the decoder side, conditional language modeling has been used to generate output sentences with the target style. In addition, output length control with conditional language modeling has been well studied (Kikuchi et al., 2016; Takeno et al., 2017; Fan et al., 2018). Our style-controllable RC relies on conditional language modeling in the decoder.

Multi-passage RC. The simplest approach is to concatenate the passages and find the answer from the concatenation, as in (Wang et al., 2017). Earlier pipelined models found a small number of relevant passages with a TF-IDF based ranker and passed them to a neural reader (Chen et al., 2017; Clark and Gardner, 2018), while more recent models have used a neural re-ranker to more accurately select the relevant passages (Wang et al., 2018; Nishida et al., 2018). Also, non-pipelined models (including ours) consider all the provided passages and find the answer by comparing scores between passages (Tan et al., 2018; Wu et al., 2018). The most recent models make a proper trade-off between efficiency and accuracy (Yan et al., 2019; Min et al., 2018). 
RC with unanswerable question identification. The previous work of (Levy et al., 2017; Clark and Gardner, 2018) outputted a no-answer score depending on the probability of all answer spans. Hu et al. (2019) proposed an answer verifier to compare an answer with the question. Sun et al. (2018) jointly learned an RC model and an answer verifier. Our model introduces a classifier on top of the question-passages reader, which is not dependent on the generated answer.

Abstractive summarization. Current state-ofthe-art models use the pointer-generator mechanism (See et al., 2017). In particular, content selection approaches, which decide what to summarize, have recently been used with abstractive models. Most methods select content at the sentence level (Hsu et al., 2018; Chen and Bansal, 2018) or the word level (Pasunuru and Bansal, 2018; Li et al., 2018; Gehrmann et al., 2018). Our model incorporates content selection at the passage level in the combined attention.

Query-based summarization has rarely been studied because of a lack of datasets. Nema et al. (2017) proposed an attentional encoder-decoder model; however, Saha et al. (2018) reported that it performed worse than BiDAF on DuoRC. Hasselqvist et al. (2017) proposed a pointer-generator based model; however, it does not consider copying words from the question.

\section{Conclusion}

This study sheds light on multi-style generative RC. Our proposed model, Masque, is based on multi-source abstractive summarization and learns multi-style answers together. It achieved stateof-the-art performance on the Q\&A task and the Q\&A + NLG task of MS MARCO 2.1 and the summary task of NarrativeQA. The key to its success is transferring the style-independent NLG capability to the target style by use of the question-passages reader and the conditional pointer-generator decoder. In particular, the capability of copying words from the question and passages can be shared among the styles, while the capability of controlling the mixture weights for the generative and copy distributions can be acquired for each style. Our future work will involve exploring the potential of our multi-style learning towards natural language understanding.

\section{References}

Lei Jimmy Ba, Ryan Kiros, and Geoffrey E. Hinton. 2016. Layer normalization. Computing Research Repository (CoRR), arXiv:1607.06450. Version 1.

Payal Bajaj, Daniel Campos, Nick Craswell, Li Deng, Jianfeng Gao, Xiaodong Liu, Rangan Majumder, Andrew McNamara, Bhaskar Mitra, Tri Nguyen, Mir Rosenberg, Xia Song, Alina Stoica, Saurabh Tiwary, and Tong Wang. 2018. MS MARCO: A human generated machine reading comprehension dataset. Computing Research Repository (CoRR), arXiv:1611.09268. Version 3.

Lisa Bauer, Yicheng Wang, and Mohit Bansal. 2018. Commonsense for generative multi-hop question answering tasks. In Empirical Methods in Natural Language Processing (EMNLP), pages 4220-4230.

Richard Caruana. 1997. Multitask learning. Machine Learning, 28(1):41-75.

Danqi Chen, Adam Fisch, Jason Weston, and Antoine Bordes. 2017. Reading wikipedia to answer opendomain questions. In Association for Computational Linguistics (ACL), pages 1870-1879.

Yen-Chun Chen and Mohit Bansal. 2018. Fast abstractive summarization with reinforce-selected sentence rewriting. In Association for Computational Linguistics (ACL), pages 675-686.

Christopher Clark and Matt Gardner. 2018. Simple and effective multi-paragraph reading comprehension. In Association for Computational Linguistics (ACL), pages 845-855.

Jacob Devlin, Ming-Wei Chang, Kenton Lee, and Kristina Toutanova. 2018. BERT: pre-training of deep bidirectional transformers for language understanding. Computing Research Repository (CoRR), arXiv:1810.04805. Version 1.

Angela Fan, David Grangier, and Michael Auli. 2018. Controllable abstractive summarization. In Workshop on Neural Machine Translation and Generation(NMT@ACL), pages 45-54.

Zhenxin Fu, Xiaoye Tan, Nanyun Peng, Dongyan Zhao, and Rui Yan. 2018. Style transfer in text: Exploration and evaluation. In Association for the Advancement of Artificial Intelligence (AAAI), pages 663-670.

Sebastian Gehrmann, Yuntian Deng, and Alexander M. Rush. 2018. Bottom-up abstractive summarization. In Empirical Methods in Natural Language Processing (EMNLP), pages 4098-4109.

Alex Graves. 2013. Generating sequences with recurrent neural networks. Computing Research Repository (CoRR), arXiv:1308.0850. Version 5.

Johan Hasselqvist, Niklas Helmertz, and Mikael Kågebäck. 2017. Query-based abstractive summarization using neural networks. arXiv, 1712.06100. 
Kaiming He, Xiangyu Zhang, Shaoqing Ren, and Jian Sun. 2016. Deep residual learning for image recognition. In Computer Vision and Pattern Recognition (CVPR), pages 770-778.

Wei He, Kai Liu, Jing Liu, Yajuan Lyu, Shiqi Zhao, Xinyan Xiao, Yuan Liu, Yizhong Wang, Hua Wu, Qiaoqiao She, Xuan Liu, Tian $\mathrm{Wu}$, and Haifeng Wang. 2018. DuReader: a chinese machine reading comprehension dataset from real-world applications. In Workshop on Machine Reading for Question Answering (MRQA@ACL), pages 37-46.

Dan Hendrycks and Kevin Gimpel. 2016. Bridging nonlinearities and stochastic regularizers with gaussian error linear units. Computing Research Repository $($ CoRR), arXiv:1606.08415. Version 2.

Wan-Ting Hsu, Chieh-Kai Lin, Ming-Ying Lee, Kerui Min, Jing Tang, and Min Sun. 2018. A unified model for extractive and abstractive summarization using inconsistency loss. In Association for Computational Linguistics (ACL), pages 132-141.

Minghao Hu, Yuxing Peng, Furu Wei, Zhen Huang, Dongsheng Li, Nan Yang, and Ming Zhou. 2018. Attention-guided answer distillation for machine reading comprehension. In Empirical Methods in Natural Language Processing (EMNLP), pages 2077-2086.

Minghao Hu, Furu Wei, Yuxing Peng, Zhen Huang, Nan Yang, and Ming Zhou. 2019. Read + Verify: Machine reading comprehension with unanswerable questions. In Association for the Advancement of Artificial Intelligence (AAAI).

Zhiting $\mathrm{Hu}$, Zichao Yang, Xiaodan Liang, Ruslan Salakhutdinov, and Eric P. Xing. 2017. Toward controlled generation of text. In International Conference on Machine Learning (ICML), pages 15871596.

Hakan Inan, Khashayar Khosravi, and Richard Socher. 2017. Tying word vectors and word classifiers: A loss framework for language modeling. In International Conference on Learning Representations (ICLR).

Sathish Reddy Indurthi, Seunghak Yu, Seohyun Back, and Heriberto Cuayáhuitl. 2018. Cut to the chase: A context zoom-in network for reading comprehension. In Empirical Methods in Natural Language Processing (EMNLP), pages 570-575.

Melvin Johnson, Mike Schuster, Quoc V. Le, Maxim Krikun, Yonghui Wu, Zhifeng Chen, Nikhil Thorat, Fernanda B. Viégas, Martin Wattenberg, Greg Corrado, Macduff Hughes, and Jeffrey Dean. 2017. Google's multilingual neural machine translation system: Enabling zero-shot translation. Transactions of the Association for Computational Linguistic (TACL), 5:339-351.
Mandar Joshi, Eunsol Choi, Daniel S. Weld, and Luke Zettlemoyer. 2017. TriviaQA: A large scale distantly supervised challenge dataset for reading comprehension. In Association for Computational Linguistics (ACL), pages 1601-1611.

Yuta Kikuchi, Graham Neubig, Ryohei Sasano, Hiroya Takamura, and Manabu Okumura. 2016. Controlling output length in neural encoder-decoders. In Empirical Methods in Natural Language Processing (EMNLP), pages 1328-1338.

Tomás Kociský, Jonathan Schwarz, Phil Blunsom, Chris Dyer, Karl Moritz Hermann, Gábor Melis, and Edward Grefenstette. 2018. The NarrativeQA reading comprehension challenge. Transactions of the Association for Computational Linguistic (TACL), 6:317-328.

Omer Levy, Minjoon Seo, Eunsol Choi, and Luke Zettlemoyer. 2017. Zero-shot relation extraction via reading comprehension. In Computational Natural Language Learning (CoNLL), pages 333-342.

Chenliang $\mathrm{Li}$, Weiran $\mathrm{Xu}, \mathrm{Si} \mathrm{Li}$, and Sheng Gao. 2018. Guiding generation for abstractive text summarization based on key information guide network. In North American Chapter of the Association for Computational Linguistics: Human Language Technologies (NAACL-HLT), pages 55-60.

Bryan McCann, Nitish Shirish Keskar, Caiming Xiong, and Richard Socher. 2018. The natural language decathlon: Multitask learning as question answering. Computing Research Repository (CoRR), arXiv:1806.08730. Version 1.

Sewon Min, Victor Zhong, Richard Socher, and Caiming Xiong. 2018. Efficient and robust question answering from minimal context over documents. In Association for Computational Linguistics (ACL), pages 1725-1735.

Preksha Nema, Mitesh M. Khapra, Anirban Laha, and Balaraman Ravindran. 2017. Diversity driven attention model for query-based abstractive summarization. In Association for Computational Linguistics (ACL), pages 1063-1072.

Kyosuke Nishida, Itsumi Saito, Atsushi Otsuka, Hisako Asano, and Junji Tomita. 2018. Retrieve-andread: Multi-task learning of information retrieval and reading comprehension. In Conference on Information and Knowledge Management (CIKM), pages 647-656.

Kishore Papineni, Salim Roukos, Todd Ward, and WeiJing Zhu. 2002. Bleu: a method for automatic evaluation of machine translation. In Association for Computational Linguistics (ACL), pages 311-318.

Ramakanth Pasunuru and Mohit Bansal. 2018. Multireward reinforced summarization with saliency and entailment. In North American Chapter of the Association for Computational Linguistics: Human Language Technologies (NAACL-HLT), pages 646-653. 
Jeffrey Pennington, Richard Socher, and Christopher D. Manning. 2014. Glove: Global vectors for word representation. In Empirical Methods in Natural Language Processing (EMNLP), pages 15321543.

Matthew E. Peters, Mark Neumann, Mohit Iyyer, Matt Gardner, Christopher Clark, Kenton Lee, and Luke Zettlemoyer. 2018. Deep contextualized word representations. In North American Chapter of the Association for Computational Linguistics: Human Language Technologies (NAACL-HLT), pages 22272237.

Alec Radford, Karthik Narasimhan, Tim Salimans, and Ilya Sutskever. 2018. Improving language understanding by generative pre-training. Technical report, OpenAI.

Alec Radford, Jeffrey Wu, Rewon Child, David Luan, Dario Amodei, and Ilya Sutskever. 2019. Language models are unsupervised multitask learners. Technical report, OpenAI.

Pranav Rajpurkar, Robin Jia, and Percy Liang. 2018. Know what you don't know: Unanswerable questions for SQuAD. In Association for Computational Linguistics (ACL), pages 784-789.

Pranav Rajpurkar, Jian Zhang, Konstantin Lopyrev, and Percy Liang. 2016. SQuAD: 100,000+ questions for machine comprehension of text. In Empirical Methods in Natural Language Processing (EMNLP), pages 2383-2392.

Siva Reddy, Danqi Chen, and Christopher D. Manning. 2018. CoQA: A conversational question answering challenge. Computing Research Repository (CoRR), arXiv:1808.07042. Version 1.

Amrita Saha, Rahul Aralikatte, Mitesh M. Khapra, and Karthik Sankaranarayanan. 2018. DuoRC: Towards complex language understanding with paraphrased reading comprehension. In Association for Computational Linguistics (ACL), pages 1683-1693.

Abigail See, Peter J. Liu, and Christopher D. Manning. 2017. Get to the point: Summarization with pointergenerator networks. In Association for Computational Linguistics (ACL), pages 1073-1083.

Rico Sennrich, Barry Haddow, and Alexandra Birch. 2016. Controlling politeness in neural machine translation via side constraints. In North American Chapter of the Association for Computational Linguistics: Human Language Technologies (NAACL$H L T)$, pages 35-40.

Minjoon Seo, Aniruddha Kembhavi, Ali Farhadi, and Hannaneh Hajishirzi. 2017. Bidirectional attention flow for machine comprehension. In International Conference on Learning Representations (ICLR).

Rupesh Kumar Srivastava, Klaus Greff, and Jürgen Schmidhuber. $2015 . \quad$ Highway networks. Computing Research Repository (CoRR), arXiv:1505.00387. Version 2.
Fu Sun, Linyang Li, Xipeng Qiu, and Yang Liu. 2018. U-Net: Machine reading comprehension with unanswerable questions. Computing Research Repository (CoRR), arXiv:1810.06638. Version 1.

Shunsuke Takeno, Masaaki Nagata, and Kazuhide Yamamoto. 2017. Controlling target features in neural machine translation via prefix constraints. In Workshop on Asian Translation (WAT@IJCNLP), pages 55-63.

Chuanqi Tan, Furu Wei, Nan Yang, Bowen Du, Weifeng Lv, and Ming Zhou. 2018. S-Net: From answer extraction to answer synthesis for machine reading comprehension. In Association for the Advancement of Artificial Intelligence (AAAI), pages 5940-5947.

Yi Tay, Anh Tuan Luu, Siu Cheung Hui, and Jian Su. 2018. Densely connected attention propagation for reading comprehension. In Advances in Neural Information Processing Systems (NeurIPS), pages 4911-4922.

Yulia Tsvetkov, Alan W. Black, Ruslan Salakhutdinov, and Shrimai Prabhumoye. 2018. Style transfer through back-translation. In Association for Computational Linguistics (ACL), pages 866-876.

Ashish Vaswani, Noam Shazeer, Niki Parmar, Jakob Uszkoreit, Llion Jones, Aidan N. Gomez, Lukasz Kaiser, and Illia Polosukhin. 2017. Attention is all you need. In Advances in Neural Information Processing Systems (NIPS), pages 6000-6010.

Shuohang Wang, Mo Yu, Xiaoxiao Guo, Zhiguo Wang, Tim Klinger, Wei Zhang, Shiyu Chang, Gerald Tesauro, Bowen Zhou, and Jing Jiang. 2018. $\mathrm{R}^{3}$ : Reinforced reader-ranker for open-domain question answering. In Association for the Advancement of Artificial Intelligence (AAAI), pages 5981-5988.

Wenhui Wang, Nan Yang, Furu Wei, Baobao Chang, and Ming Zhou. 2017. Gated self-matching networks for reading comprehension and question answering. In Association for Computational Linguistics (ACL), pages 189-198.

Hua Wu, Haifeng Wang, Sujian Li, Wei He, Yizhong Wang, Jing Liu, Kai Liu, and Yajuan Lyu. 2018. Multi-passage machine reading comprehension with cross-passage answer verification. In Association for Computational Linguistics (ACL), pages 19181927.

Caiming Xiong, Victor Zhong, and Richard Socher. 2017. Dynamic coattention networks for question answering. In International Conference on Learning Representations (ICLR).

Ming Yan, Jiangnan Xia, Chen Wu, Bin Bi, Zhongzhou Zhao, Ji Zhang, Luo Si, Rui Wang, Wei Wang, and Haiqing Chen. 2019. A deep cascade model for multi-document reading comprehension. In Association for the Advancement of Artificial Intelligence (AAAI). 
Zhilin Yang, Peng Qi, Saizheng Zhang, Yoshua Bengio, William W. Cohen, Ruslan Salakhutdinov, and Christopher D. Manning. 2018. HotpotQA: A dataset for diverse, explainable multi-hop question answering. In Empirical Methods in Natural Language Processing (EMNLP), pages 2369-2380.

Dani Yogatama, Cyprien de Masson d'Autume, Jerome Connor, Tomás Kociský, Mike Chrzanowski, Lingpeng Kong, Angeliki Lazaridou, Wang Ling, Lei Yu, Chris Dyer, and Phil Blunsom. 2019. Learning and evaluating general linguistic intelligence. Computing Research Repository (CoRR), arXiv:1901.11373. Version 1.

Adams Wei Yu, David Dohan, Minh-Thang Luong, Rui Zhao, Kai Chen, Mohammad Norouzi, and Quoc V. Le. 2018. QANet: Combining local convolution with global self-attention for reading comprehension. In International Conference on Learning Representations (ICLR). 